\title{
Crystal-to-Crystal Transition of Ultrasoft Colloids under Shear
}

\author{
J. Ruiz-Franco, ${ }^{1}$ J. Marakis, ${ }^{2,3}$ N. Gnan, ${ }^{1,4}$ J. Kohlbrecher, ${ }^{5}$ M. Gauthier, ${ }^{6}$ M. P. Lettinga, ${ }^{7,8}$ \\ D. Vlassopoulos, ${ }^{2,3, *}$ and E. Zaccarelli ${ }^{1,4, \dagger}$ \\ ${ }^{1}$ Dip. Di Fisica, Sapienza Università di Roma, Piazzale A. Moro 5, 00185 Rome, Italy \\ ${ }^{2}$ Foundation for Research and Technology-Hellas (FORTH), Institute of Electronic Structure and Laser (IESL), \\ 100 N. Plastira Street, GR-70013 Heraklion, Greece \\ ${ }^{3}$ Department of Materials Science and Technology, University of Crete, P.O. Box 2208, GR-71003 Heraklion, Greece \\ ${ }^{4}$ CNR Institute of Complex Systems, Uos Sapienza, Piazzale A. Moro 5, 00185 Rome, Italy \\ ${ }^{5}$ Laboratory for Neutron Scattering and Imaging, Paul Scherrer Institute, 5232 Villigen PSI, Switzerland \\ ${ }^{6}$ Department of Chemistry, University of Waterloo, Waterloo, Ontario N2L 3G1, Canada \\ ${ }^{7}$ Laboratory for Soft Matter and Biophysics, KU Leuven, Celestijnenlaan 200D, B-3001 Leuven, Belgium \\ ${ }^{8}$ Institute of Complex Systems (ICS-3), Forschungszentrum Jülich, 52425 Jülich, Germany
}

(Received 16 October 2017; published 16 February 2018)

\begin{abstract}
Ultrasoft colloids typically do not spontaneously crystallize, but rather vitrify, at high concentrations. Combining in situ rheo-small-angle-neutron-scattering experiments and numerical simulations we show that shear facilitates crystallization of colloidal star polymers in the vicinity of their glass transition. With increasing shear rate well beyond rheological yielding, a transition is found from an initial bcc-dominated structure to an fcc-dominated one. This crystal-to-crystal transition is not accompanied by intermediate melting but occurs via a sudden reorganization of the crystal structure. Our results provide a new avenue to tailor colloidal crystallization and the crystal-to-crystal transition at the molecular level by coupling softness and shear.
\end{abstract}

DOI: 10.1103/PhysRevLett.120.078003

Concentrated suspensions of Brownian spheres are known to undergo crystallization and/or a glass transition, depending on their size polydispersity and interaction potential [1]. With respect to hard spheres, quiescent crystallization in dense suspensions of soft colloids is in general more complicated, due to shape fluctuations and adjustment $[2,3]$. Whereas microgel-based particles crystallize at roughly the same packing fraction as hard spheres [4-6], hairy particles may do so at larger concentrations, depending of the relative core-to-grafted arm size ratio or the rate of arm exchange in the case of micelles [7-10]. Particles with small cores and long hairs, such as star polymers, cannot crystallize easily because arm fluctuations delay or hinder this process $[11,12]$, despite the opposite expectations due to enhanced osmotic pressure [3]. The slowdown of the nucleation process can also be attributed to interpenetration and clustering, which may act as an effective polydispersity suppressing crystallization [13]. It is therefore common for soft colloids to become kinetically trapped in metastable states [14]. Colloidal glasses may crystallize eventually over time; i.e., thermodynamic equilibrium is reached,

Published by the American Physical Society under the terms of the Creative Commons Attribution 4.0 International license. Further distribution of this work must maintain attribution to the author(s) and the published article's title, journal citation, and DOI. irrespectively of softness $[11,12,15]$. The action of an external stimulus, such as shear flow, can promote either formation or melting of ordered states, depending on its rate and strength $[16,17]$. Hence, the delicate interplay between interparticle forces and hydrodynamic interactions provides the conditions for achieving and tuning colloidal crystallization or dynamic arrest [16-26].

The ability of shear to induce crystal formation in soft colloids is significant and well documented [27-32]. Depending on the rate of applied oscillatory or steady shear, a rich variety of crystal phases can be formed, which are often able to sustain large deformations [28-32]. However, promoting crystallization in sheared glassy or jammed systems is challenging since their original microstructures are nonequilibrium states that may undergo phase or layering transitions while deformation of soft particles is possible [33-35]. Ultrasoft colloidal stars, for which the size and number of arms determine the interactions between particles $[9,36]$, display a very rich glassy phenomenology $[35,37]$. At the level of particle microstructure, the interpenetration of the arms is primarily responsible for their complex rheological behavior [37-39], implying that shear could promote crystal formation of stars via their cooperative rearrangement, which is mediated by arm disengagement. This avenue to crystallization for hairy ultrasoft colloids is yet to be explored.

In addition to shear-induced order, order-to-order transitions under the influence of an external stimulus are 
ubiquitous in colloidal systems. In particular, microgels have been found to undergo a crystal-to-crystal transition upon changing temperature in equilibrium [40] and in the presence of an electric field [41]. For block copolymer micelles $[27,29,31,42]$ and microgel dumbbells [43] such transitions have been observed with increasing shear rate. It has been argued that the crystal-to-crystal transition occurs via twostep transformations, accompanied by the formation of an intermediate fluid phase $[29,40,43]$, which favors local rearrangements and subsequent recrystallization. Such intermediate melting was thus suggested to be a generic mechanism for the occurrence of crystal-to-crystal transitions [44]. However, whether such a transition and mechanism hold for ultrasoft colloids is an important open question.

In this Letter we investigate the consequences of an imposed shear flow on the crystallization of colloidal stars in the vicinity of their glass transition by means of in situ rheo-small-angle-neutron-scattering (rheo-SANS) experiments and molecular dynamics simulations (MD). We find that shear promotes crystallization, both under oscillatory (experiments) and steady (simulations) conditions. Moreover, we provide unambiguous evidence of a crystal-to-crystal transition under shear. Results from measured and calculated diffraction patterns, which are in good agreement, suggest a two-step process. At first the fluid forms a bcc-like crystal (first step), which later transforms into a fcc-like one (second step) through a sudden change in the crystal structure. Differently from previous observations [29,40,43], we do not find evidence of an intermediate liquid phase between the two crystals.

We investigate 1,4-polybutadiene stars with functionality $f=203$ arms and arm molar mass of $30500 \mathrm{~g} / \mathrm{mol}$ [45]. The hydrodynamic radius in toluene (an athermal solvent) is $45 \mathrm{~nm}$ and the overlap concentration $c^{*}=27 \mathrm{mg} / \mathrm{ml}$. The softness of the stars can be quantified by the softness parameter $\mathrm{SP}=0.11 \quad[35,46]$, as described in the Supplemental Material [47]. We study different concentrations $\left(2 c^{*}-2.2 c^{*}\right)$, corresponding to a range of packing fractions $\eta \approx 0.15-0.167$ [9], in the vicinity of the mestastable glassy regime shown in the phase diagram of Fig. 1(a). Samples do not crystallize in the absence of an external field for the investigated time (1 day). The rheological characterization was performed by means of dynamic oscillatory measurements using a sensitive stresscontrolled rheometer operating in the strain-controlled mode (see Fig. S1 of Ref. [47]). Rheo-SANS measurements were carried out at the Swiss spallation neutron source (SINQ) of the Paul Scherrer Institut in Villigen, Switzerland. The rheo-SANS setup combined SANS and a stress-controlled rheometer, which offered the possibility of performing measurements in the radial (velocityvorticity, $\vec{v}, \vec{v} \times \vec{\nabla} \vec{v}$ ) and tangential (velocity gradientvorticity, $\vec{\nabla} \vec{v}, \vec{v} \times \vec{\nabla} \vec{v}$ ) planes, as illustrated in Fig. 1(b). Further details are provided in Ref. [47].

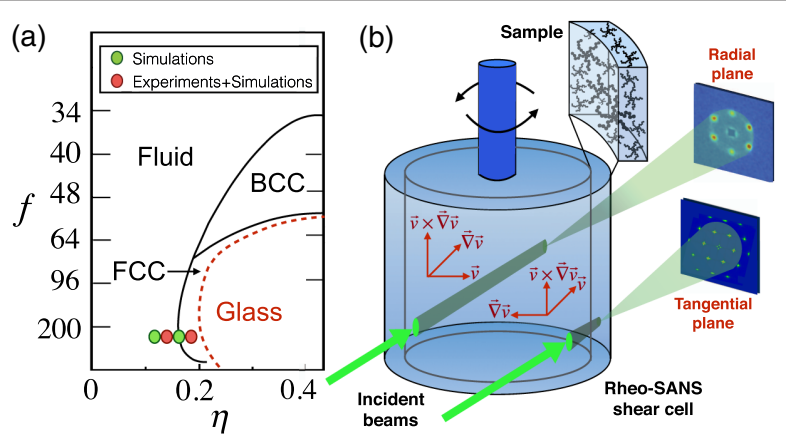

FIG. 1. (a) Theoretical state diagram of star polymers $[9,14]$ in the $(f, \eta)$ plane. State points investigated in this work are marked with symbols. (b) Schematic illustration of the rheo-SANS experiments and measured diffraction patterns in the radial and tangential plane. See text for details.

The experimental investigations are complemented by numerical simulations of particles interacting via a coarsegrained, ultrasoft effective potential, which mimics the interactions between star polymers $[36,48]$. We perform MD simulations for $N=2000$ stars with functionality $f=203$ at different packing fractions [Fig. 1(a)]. We use a steady shear protocol at fixed shear rate $\dot{\gamma}$ complemented by Lees-Edwards boundary conditions [49] and a dissipative particle dynamics thermostat [50-52]. To quantify crystallization, we calculate local and averaged bond order parameter distributions [53,54], assigning solidlike nature to each particle [55] and also distinguishing between different crystal structures [56]. We also monitor the fraction of solidlike particles and define a crystallization time $t_{X}$ when this fraction reaches $20 \%$ [57]. Numerical results are averaged over five independent realizations.

To compare experimental and numerical results obtained under different shear protocols, we use the Péclet number $\mathrm{Pe}=\dot{\gamma} \tau_{B}$, where $\tau_{B}$ is the Brownian time defined in terms of the self-diffusion coefficient at infinite dilution. With this definition Péclet numbers vary in the range $10^{-5} \lesssim$ $\mathrm{Pe} \lesssim 10^{-1}$ (see Ref. [47]). The experimental shear rate is $\dot{\gamma}=\gamma_{0} \omega$, with $\gamma_{0}$ the strain amplitude and $\omega$ the frequency. In both experiments and simulations we also calculate the degree of order parameter (DOO) which captures the increase of the intensity in the diffraction patterns associated with the growth of crystalline order in the system. More details are provided in Ref. [47].

For the investigated packing fractions, the system at rest is a metastable liquid or glass as revealed by linear viscoelastic measurements (see Fig. S1 of Ref. [47]), reflecting the proximity of the studied state points to the fluid-crystal (fcc) boundary predicted theoretically $[9,11]$. In all cases the samples were sheared at rates corresponding to the solidlike region of the linear viscoelastic spectrum (Fig. S1 of Ref. [47]). To monitor the crystallization process, we report in Fig. 2 the DOO for the amorphous structure (fluid or glass) to crystal transition observed in [Fig. 2(a)] experiments and [Fig. 2(b)] simulations, 

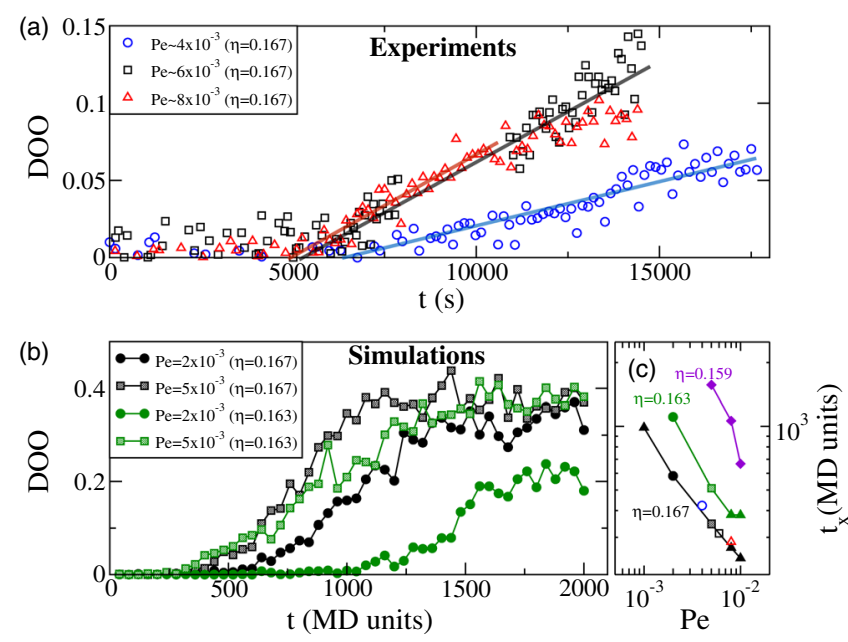

FIG. 2. Degree of ordering (DOO) versus time calculated for experiments (a) and simulations (b). In (a) the shear parameters are $\omega=10 \mathrm{rad} / \mathrm{s}$ and $\gamma_{0}=11.5 \%$ (squares), $\omega=5 \mathrm{rad} / \mathrm{s}$ and $\gamma_{0}=15 \%$ (circles), and $\omega=10 \mathrm{rad} / \mathrm{s}$ and $\gamma_{0}=15 \%$ (triangles). Lines are guides to the eye to highlight the onset of crystallization. (c) Nucleation time $t_{X}$ as a function of Pe for simulations at different packing fractions (filled symbols) and for experiments (scaled by an arbitrary factor) at $\eta=0.167$ [open symbols as in panel (a)].

showing the same qualitative trends: (i) there is an induction time for crystallization to occur; (ii) at the same star packing fraction crystallization is faster and more pronounced with increasing Pe; (iii) under the same shear conditions, an increase of $\eta$ facilitates and speeds up crystallization. These features are also evident in Fig. 2(c), where the crystallization time $t_{X}$ is reported as a function of Pe for different values of $\eta$. It is also found experimentally that frequency has a stronger influence on the DOO [Fig. 2(a)], and thus on the nucleation time, with respect to strain amplitude. These results confirm earlier results for hard sphere systems [18,21,22,58,59], suggesting that in general large enough shear rates are needed in order to induce crystallization. Some quantitative differences between simulations and experiments (the former being more sensitive to shear rate) are attributed to the different protocols used.

The calculation of bond order parameters $[54,56]$ in simulations reveals that the fluid-to-crystal transition in most cases, and always for large enough Pe, gives rise to a fcc-like crystal (see Fig. S2 of Ref. [47]). While for very low values of $\mathrm{Pe}\left(\mathrm{Pe} \lesssim 10^{-3}\right)$ no crystallization takes place, for intermediate values of $\dot{\gamma}$ we observe a two-step process: at first a transition occurs from the fluid to a bcc-like crystal, later followed by a second transition to a fcc- or hcp-like crystal. Both transitions are accompanied by clear discontinuities in the energy of the system (Fig. S3 of Ref. [47]). A crystal-to-crystal transition is found for $0.159 \leq \eta \leq 0.167$ at sufficiently small Pe. On decreasing the packing fraction the crystal-to-crystal transition is observed by increasing Pe.
Such a behavior is also found in experiments at $\eta=$ 0.167 upon continuous application of strain amplitude from $0.1 \%$ to $300 \%$ (within $600 \mathrm{~s}$ ) with a frequency $\omega=5 \mathrm{rad} / \mathrm{s}$, as reported in Fig. 3(a): a transition from amorphous glassto-crystal takes place at $\mathrm{Pe} \sim 1.4 \times 10^{-4}\left(\gamma_{0}=0.5 \%\right)$, followed by a crystal-to-crystal transition at strain amplitudes higher than $120 \%\left(\mathrm{Pe} \gtrsim 3.3 \times 10^{-2}\right)$, well above rheological yielding. A crystal-to-crystal transition was only observed for $\omega=5 \mathrm{rad} / \mathrm{s}$ and not for larger frequencies, suggesting that not too high shear rates are required to induce the first transition to an intermediate crystal structure. Although it is not straightforward to compare parameters obtained with different shear protocols, these findings are in qualitative agreement with simulations.

From the radial rheo-SANS diffraction patterns shown in Fig. 3(a) we can speculate that a transition takes place between two hexagonal ordered structures oriented along different directions. To verify this interpretation, we rely on numerical simulations and calculate diffraction patterns from the particle coordinates [60]. In Figs. 3(b1) and 3(b2) we report the diffraction patterns in the radial direction of the first and second crystal, respectively. The numerical results are again in good agreement with the experimental SANS patterns [Fig 3(a)], despite the difference in the used shear protocol. To visualize the two (fluid-to-crystal and crystal-to-crystal) transitions, movies of simulations are presented in Ref. [47], while snapshots of the two crystal structures in the tangential plane are reported in Figs. 3(c1) and 3(c2). After completing the first step [Fig. 3(b1)], the crystal is organized into two different layers oriented orthogonally both to the vorticity and to the velocity gradient directions due to the bcc geometry, while after the second step the layers reorganize and become orthogonal with respect to the gradient direction only [Fig. 3(b2)]. These features are clearly evidenced by looking at the calculated density profiles along different directions, respectively, for bcc [Fig. 3(d1)] and fcc or hcp particles [Fig. 3(d2)]. We observe oscillations in the density in both the velocity-gradient and vorticity directions after the first step. However, after the second step, a flat profile is observed for the vorticity axis, while oscillations survive in the $\vec{\nabla} \vec{v}$ direction. The layers of fcc particles are only orthogonal to $\vec{\nabla} \vec{v}$ at all times. Figure 3(d2) also shows that an enhancement of oscillations along the $\vec{\nabla} \vec{v}$ axis after the second step for fcc-ordered particles is associated with a decrease of the same oscillation for bcc-ordered ones. These features clearly indicate that the formation of a bcc lattice is responsible for the peculiar structure observed in the first step. Indeed, the layering orthogonal to the vorticity is completely lost once these particles reorganize into an fcc lattice, giving rise to the layers commonly observed in other shear-induced experiments $[29,34,42,58]$.

To connect our findings with previous observations of crystal-to-crystal transformations, we investigate whether 

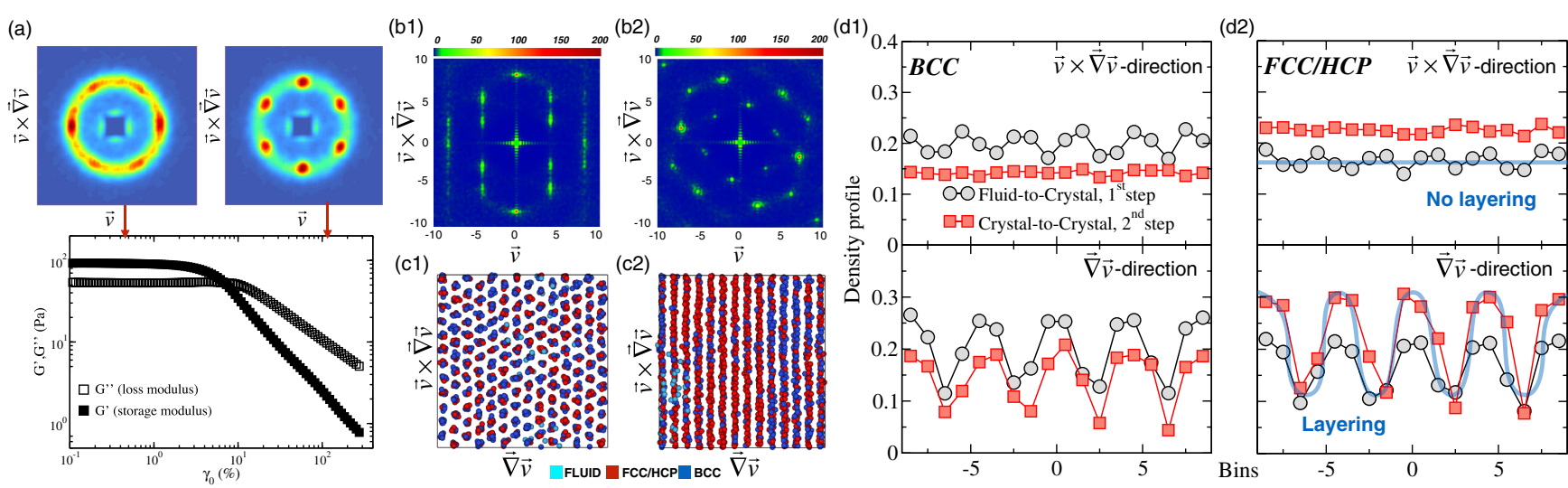

FIG. 3. (a) SANS diffraction patterns in the radial direction and strain amplitude sweep depicting the measured $G^{\prime}$ (storage modulus) $G^{\prime \prime}$ (loss modulus) for stars at $\eta=0.167$. The flipping between the two crystals was observed for $\omega=5 \mathrm{rad} / \mathrm{s}$ in a range of strain amplitude $(0.1 \%-300 \%)$, amounting to a range in Péclet numbers $\left(0-8 \times 10^{-2}\right)$. Vertical arrows indicate the strain values at which the images were extracted. (b)-(d) Numerical results for simulations at $\eta=0.167$ and $\mathrm{Pe}=2 \times 10^{-3}$. Radial diffraction patterns after the first step (b1) and after the second step (b2). System snapshots in the tangential view after the first step (c1) and after the second step (c2). Here, the size of the particles is reduced to help visualization. Density profiles measured for bcc (d1) and fcc or hcp (d2) particles in the vorticity direction (upper panels) and gradient direction (lower panels). Continuous lines are drawn to indicate the behavior of the density profiles in the absence (flat line) or in the presence (periodic curve) of layers of particles.

in our system there is evidence of intermediate melting, at least locally, which could help the (re)organization into a different lattice. To this aim we monitor the fraction of particles of each species (liquid, fcc, bcc, hcp) during the second step, finding that melting does not occur during the bcc- to fcc-like transition (see Fig. S4 of Ref. [47]). This constitutes a striking difference with respect to the case of thermoresponsive microgels studied in Ref. [40] and may be attributed in part to the different protocol used in that work, where the transition was induced by varying the temperature, rather than by shear. On the other hand, Refs. [29,43] reported intermediate melting in the presence of shear, without notable soft particle deformation. For the star polymers under shear studied in the present work, the reorganization of the crystal lattice between two competing structures occurs without intermediate melting even at the local level. Instead, we observe a sudden change, i.e., a "flipping," between the bcc and fcc lattice, which provides an alternative mechanism to realize a crystal-to-crystal transition in this system. These findings are linked to the peculiar nature of star polymers, which allows for a direct transformation between two crystals, thanks to their ultrasoft interactions. Indeed, according to the theoretical phase diagram [Fig. 1(a)], for the studied state points the system is approaching a glass transition but its underlying equilibrium state is the fcc crystal. Being dominated by Yukawalike repulsions at low packing fractions, the free energy difference between the fcc and bcc structure is very small [61]. Thus, the competition between these two crystalline structures, which is influenced by Pe, determines the final state of the sheared system. Based on our results we suggest that at high enough Pe the system experiences a fluid-tocrystal transition directly into the fcc crystal due to the large rearrangements induced by shear. On the other hand, at lower Pe shearing is not strong enough and the system is only able to complete the crystallization process in two steps, by first attaining an intermediate (metastable) bcc-like state and then reaching a state comprising a mixture of bcc and fcc structures. This is confirmed by the fact that at even lower Pe crystallization is not observed, whereas the threshold value of Pe to achieve a two-step crystallization increases with decreasing packing fraction. Importantly, once the second step is reached, the crystal does not melt upon shear cessation, but remains stable over time in both experiments and simulations. We stress that the final structure with layers parallel to the flow is in agreement with previous studies of shear-induced crystallization [29,34,42,58]. However, the intermediate structure occurring after the first step and the mechanism behind the crystal-to-crystal transition are novel features of the present study, which are attributed to the ultrasoftness of colloidal star polymers.

In summary, the application of shear induces crystallization of ultrasoft star polymer suspensions at packing fractions in the vicinity of the glass line. The good agreement between experiments and simulations, despite the different shear protocol used, strongly supports the generality of the results. In most cases, a fluid-to-crystal transition under shear is found, which is facilitated by increasing Pe and increasing the packing fraction. However, for $0.159 \leq \eta \leq 0.167$ there exists an intermediate range of Pe where stars undergo a distinct crystal-to-crystal transition. The transition consists of a transformation from a bcc-dominated to an fcc-dominated crystal, which occurs via a flipping of the crystal structure and not by an intermediate melting, differently from previous studies. Our results indicate that the combination of shear and softness is important for shedding light on the fundamental physics underlying phase transitions, as well as tailoring the organization of soft materials with 
desired properties. To this end, the tunable softness of star polymers is very valuable and future directions will include the control and manipulation of crystal-to-crystal transitions in different regions of the phase diagram, changing both functionality and packing fractions.

We are grateful to E. Stiakakis for help with sample preparation and characterization and C. N. Likos for enlightening discussions. J. R.-F., D. V., and E.Z. acknowledge support from ETN-COLLDENSE (H2020-MCSA-ITN2014, Grant No. 642774); J. M. and D. V. acknowledge support from the Greek General Secretariat for Research and Technology in the framework of the program Thalis (Project METAASSEMBLY); D. V. acknowledges support from the Aage og Johanne Louis-Hansen Foundation; M. G. acknowledges support from the Natural Sciences and Engineering Research Council of Canada; N. G. and E. Z. acknowledge support from European Research Council Consolidator Grant No. 681597 MIMIC.

J. R.-F. and J. M., contributed equally to this work.

*Corresponding author.

dvlasso@iesl.forth.gr

${ }^{\dagger}$ Corresponding author. emanuela.zaccarelli@cnr.it

[1] P. Pusey, in Liquids, Freezing and the Glass Transition, Proceedings of the Les Houches Summer School, Session number LI, edited by J.P. Hansen, D. Levesque, and J. Zinn-Justin (North-Holland Amsterdam, 1991), pp. 763-942.

[2] J. Zhang, P. M. Lettinga, J. K. G. Dhont, and E. Stiakakis, Phys. Rev. Lett. 113, 268303 (2014).

[3] T. Witten, P. Pincus, and M. Cates, Europhys. Lett. 2, 137 (1986).

[4] H. Senff and W. Richtering, J. Chem. Phys. 111, 1705 (1999).

[5] L. A. Lyon, J. D. Debord, S. B. Debord, C. D. Jones, J. G. McGrath, and M. J. Serpe, J. Phys. Chem. B 108, 19099 (2004).

[6] Y. Lu and M. Ballauff, Prog. Polym. Sci. 36, 767 (2011).

[7] G. A. McConnell, A. P. Gast, J. S. Huang, and S. D. Smith, Phys. Rev. Lett. 71, 2102 (1993).

[8] F. Puaud, T. Nicolai, E. Nicol, L. Benyahia, and G. Brotons, Phys. Rev. Lett. 110, 028302 (2013).

[9] M. Watzlawek, C. N. Likos, and H. Löwen, Phys. Rev. Lett. 82, 5289 (1999).

[10] I. W. Hamley, Curr. Opin. Colloid Interface Sci. 5, 341 (2000).

[11] E. Stiakakis, A. Wilk, J. Kohlbrecher, D. Vlassopoulos, and G. Petekidis, Phys. Rev. E 81, 020402 (2010).

[12] A. N. Rissanou, M. Yiannourakou, I. G. Economou, and I. A. Bitsanis, J. Chem. Phys. 124, 044905 (2006).

[13] S. Gupta, M. Camargo, J. Stellbrink, J. Allgaier, A. Radulescu, P. Lindner, E. Zaccarelli, C. N. Likos, and D. Richter, Nanoscale 7, 13924 (2015).

[14] G. Foffi, F. Sciortino, P. Tartaglia, E. Zaccarelli, F. Lo Verso, L. Reatto, K. Dawson, and C. N. Likos, Phys. Rev. Lett. 90, 238301 (2003).
[15] E. Zaccarelli, C. Valeriani, E. Sanz, W. C. K. Poon, M. E. Cates, and P. N. Pusey, Phys. Rev. Lett. 103, 135704 (2009).

[16] H. Löwen, J. Phys. Condens. Matter 13, R415 (2001).

[17] J. Vermant and M. Solomon, J. Phys. Condens. Matter 17, R187 (2005).

[18] P. Holmqvist, M. P. Lettinga, J. Buitenhuis, and J. K. Dhont, Langmuir 21, 10976 (2005).

[19] A. Imhof, A. Van Blaaderen, and J. Dhont, Langmuir 10, 3477 (1994).

[20] T. Besseling, M. Hermes, A. Fortini, M. Dijkstra, A. Imhof, and A. van Blaaderen, Soft Matter 8, 6931 (2012).

[21] B. J. Ackerson and P. N. Pusey, Phys. Rev. Lett. 61, 1033 (1988).

[22] B. J. Ackerson, J. Rheol. 34, 553 (1990).

[23] N. Duff and D. J. Lacks, Phys. Rev. E 75, 031501 (2007).

[24] N. Koumakis, A. Schofield, and G. Petekidis, Soft Matter 4, 2008 (2008).

[25] K. Mortensen, E. Theunissen, R. Kleppinger, K. Almdal, and H. Reynaers, Macromolecules 35, 7773 (2002).

[26] Y. L. Wu, D. Derks, A. van Blaaderen, and A. Imhof, Proc. Natl. Acad. Sci. U.S.A. 106, 10564 (2009).

[27] J. Jiang, C. Burger, C. Li, J. Li, M. Y. Lin, R. H. Colby, M. H. Rafailovich, and J. C. Sokolov, Macromolecules 40, 4016 (2007).

[28] T. M. Slawecki, C. J. Glinka, and B. Hammouda, Phys. Rev. E 58, R4084 (1998).

[29] C. R. López-Barrón, N. J. Wagner, and L. Porcar, J. Rheol. 59, 793 (2015).

[30] F. Molino, J.-F. Berret, G. Porte, O. Diat, and P. Lindner, Eur. Phys. J. B 3, 59 (1998).

[31] G. A. McConnell, M. Y. Lin, and A. P. Gast, Macromolecules 28, 6754 (1995).

[32] A. Nikoubashman, G. Kahl, and C. N. Likos, Soft Matter 8, 4121 (2012).

[33] J. R. Stokes and W. J. Frith, Soft Matter 4, 1133 (2008).

[34] F. Khabaz, T. Liu, M. Cloitre, and R. T. Bonnecaze, Phys. Rev. Fluids 2, 093301 (2017).

[35] D. Vlassopoulos and M. Cloitre, Curr. Opin. Colloid Interface Sci. 19, 561 (2014).

[36] C. N. Likos, H. Löwen, M. Watzlawek, B. Abbas, O. Jucknischke, J. Allgaier, and D. Richter, Phys. Rev. Lett. 80, 4450 (1998).

[37] M. E. Helgeson, N. J. Wagner, and D. Vlassopoulos, J. Rheol. 51, 297 (2007).

[38] D. Vlassopoulos, G. Fytas, T. Pakula, and J. Roovers, J. Phys. Condens. Matter 13, R855 (2001).

[39] M. Kapnistos, A. N. Semenov, D. Vlassopoulos, and J. Roovers, J. Chem. Phys. 111, 1753 (1999).

[40] Y. Peng, F. Wang, Z. Wang, A. M. Alsayed, Z. Zhang, A. G. Yodh, and Y. Han, Nat. Mater. 14, 101 (2015).

[41] P. S. Mohanty, P. Bagheri, S. Nöjd, A. Yethiraj, and P. Schurtenberger, Phys. Rev. X 5, 011030 (2015).

[42] E. Eiser, F. Molino, G. Porte, and O. Diat, Phys. Rev. E 61, 6759 (2000).

[43] F. Chu, N. Heptner, Y. Lu, M. Siebenbürger, P. Lindner, J. Dzubiella, and M. Ballauff, Langmuir 31, 5992 (2015).

[44] E. Sanz and C. Valeriani, Nat. Mater. 14, 15 (2015).

[45] M. Gauthier and A. Munam, Macromolecules 43, 3672 (2010).

[46] M. Daoud and J. Cotton, J. Phys. (Paris) 43, 531 (1982). 
[47] See Supplemental Material at http://link.aps.org/ supplemental/10.1103/PhysRevLett.120.078003 for materials characterization, rheometric data, simulation methods and further analysis of shear-induced effects.

[48] C. N. Likos, Phys. Rep. 348, 267 (2001).

[49] A. Lees and S. Edwards, J. Phys. C 5, 1921 (1972).

[50] J. Zausch, J. Horbach, M. Laurati, S. U. Egelhaaf, J. M. Brader, T. Voigtmann, and M. Fuchs, J. Phys. Condens. Matter 20, 404210 (2008).

[51] A. Nicolas, J.-L. Barrat, and J. Rottler, Phys. Rev. Lett. 116, 058303 (2016).

[52] E. Peters, Europhys. Lett. 66, 311 (2004).

[53] P. J. Steinhardt, D. R. Nelson, and M. Ronchetti, Phys. Rev. B 28, 784 (1983).
[54] W. Lechner and C. Dellago, J. Chem. Phys. 129, 114707 (2008)

[55] P. Pusey, E. Zaccarelli, C. Valeriani, E. Sanz, W. C. Poon, and M. E. Cates, Phil. Trans. R. Soc. A 367, 4993 (2009).

[56] J. Russo and H. Tanaka, Sci. Rep. 2, 505 (2012).

[57] C. Valeriani, E. Sanz, P. N. Pusey, W. C. Poon, M. E. Cates, and E. Zaccarelli, Soft Matter 8, 4960 (2012).

[58] R. Blaak, S. Auer, D. Frenkel, and H. Löwen, Phys. Rev. Lett. 93, 068303 (2004).

[59] D. Richard and T. Speck, Sci. Rep. 5 (2015).

[60] A. Metere, P. Oleynikov, M. Dzugutov, and S. Lidin, Soft Matter 12, 8869 (2016).

[61] M. O. Robbins, K. Kremer, and G. S. Grest, J. Chem. Phys. 88, 3286 (1988). 